\title{
De novo transcriptome assembly and analysis to identify potential gene targets for RNAi-mediated control of the tomato leafminer (Tuta absoluta)
}

Roberto de A. Camargo ${ }^{1,2}$, Roberto H. Herai ${ }^{3,4}$, Luana N. Santos ${ }^{5}$, Flavia M M Bento ${ }^{1,2}$, Joni E. Lima', Henrique Marques-Souza ${ }^{5}$ and Antonio Figueira ${ }^{1 *}$

\begin{abstract}
Background: Providing double-stranded RNA (dsRNA) to insects has been proven to silence target genes, and this approach has emerged as a potential method to control agricultural pests by engineering plants to express insect dsRNAs. A critical step of this technology is the screening of effective target genes essential for insect development and/or survival. The tomato leafminer (Tuta absoluta Meyrick) is a major Solanum lycopersicum (tomato) pest that causes significant yield losses and has recently invaded Europe, from where it is spreading at an alarming rate. To explore RNA interference (RNAi) against T. absoluta, sequence information on potential target genes is necessary, but only a few sequences are available in public databases.

Results: We sequenced six libraries from RNA samples from eggs, adults, and larvae at four stages, obtaining an overall total of around 245 million reads. The assembled T. absoluta transcriptome contained 93,477 contigs with an average size of 1,574 bp, $59.8 \%$ of which presented positive Blast hits, with 19,995 (21.4\%) annotated by gene ontology. From the transcriptome, most of the core genes of the RNAi mechanism of Lepidoptera were identified indicating the potential suitability of T. absoluta for gene silencing. No contigs displayed significant similarity with a RNA-dependent RNA Polymerase. Genes from the juvenile hormone and ecdysteroid biosynthetic pathways were identified, representing potential target genes for systemic silencing. Comparisons of transcript profiles among stages revealed 1,577 genes differentially expressed at earlier larval stages, from which potential gene targets were identified. Five of these genes were evaluated using in vitro transcribed dsRNA absorbed by tomato leaflets, which were fed to $1^{\text {st }}$ instar T. absoluta larvae, resulting in significant reduction of larval body weight while exhibiting significant knockdown for three of the genes.
\end{abstract}

Conclusions: The transcriptome we generated represents a valuable genomic resource for screening potential gene targets that affect the development or survival of T. absoluta larvae. Five novel genes that showed greater expression at the $1^{\text {st }}$ larval stage were demonstrated to be effective potential RNAi targets by reducing larval weight and can be considered good candidates for use in RNAi-mediated crop protection.

Keywords: Gelechiidae, Gene silencing, Hormone synthesis, Lepidoptera, RNA-seq, Solanaceae

\footnotetext{
* Correspondence: figueira@cena.usp.br

${ }^{1}$ Centro de Energia Nuclear na Agricultura, Universidade de São Paulo, Av.

Centenário, 303, CP 96, Piracicaba, SP 13400-970, Brazil

Full list of author information is available at the end of the article
} 


\section{Background}

Since the discovery that providing double-stranded RNA (dsRNA) to a wide variety of organisms, including insects, can induce RNA interference (RNAi), this method has become a common tool for functional genomic studies, particularly in non-model systems [1, 2]. Early studies used microinjection to deliver dsRNA into insect bodies, but the demonstration that dsRNA uptake through ingestion was sufficient to knock target genes down opened the possibility of applying this approach on a larger scale [2]. The potential application of RNAi for controlling agricultural insect pests soon became evident [3]. Application of dsRNA by crop spraying resembles current insecticide delivery methods, but RNA production costs and stability with current technology may restrict its efficacy [4]. However, the demonstration that transgenic plants modified to express insect dsRNA can successfully control target pests $[5,6]$ raised the possibility of developing RNAi-mediated crop protection [3, 4, 7]. Transgenic plants expressing dsRNA matching specific insect target genes have been demonstrated to control Lepidoptera, Coleoptera and Hemiptera agricultural pests [4], including Helicoverpa armigera in cotton [8] and tobacco [9, 10], Diabrotica virgifera virgifera in maize [5], Nilaparvata lugens in rice [11], Myzus persicae in Nicotiana benthamiana and Arabidopsis thaliana [12], and Sitobion avenae in wheat [13].

A critical requisite of this technology is the availability of a large number of potential target gene sequences to be screened for effectiveness [14]. Given that agricultural insect pests are generally non-model systems, little genomic information is available for most of them. Originally, cDNA libraries were used to screen for insect target genes [5], but the advent of massive RNA sequencing by Next-Generation Sequencing platforms has enabled the reconstruction of almost full transcriptomes under any biological condition for non-model organisms [15]. This approach to identifying potential gene targets for silencing has been successfully used for various agricultural pests, including the eastern corn borer Ostrinia furnacalis [16], the aphid Sitobion avenae [17], the beet armyworm Spodoptera exigua [18], the brown planthopper $N$. lugens [19], and the cotton boll weevil Anthonomus grandis [20], to name a few.

In addition, a full transcriptome from insects can be used to reveal genes of the core mechanism of RNAi, which is mostly conserved among Eukaryotes but tends to exhibit peculiarities among taxa. The basic repertoire of genes includes those encoding transmembrane channel proteins (e.g. SID-1) involved in dsRNA uptake and systemic spread, nucleases such as Dicer and Dicer-like that cleave dsRNAs into small interfering RNAs, and those belonging to the RNAinduced silencing complex (or RISC), including the Argonaute protein family, that recognizes dsRNA and degrades target mRNA, and other factors involved in RISC assembly, such as $R 2 D 2$, and putative RNA helicases such as Armitage and SpindleE [1,21]. A critical factor for efficient RNAi in insects is the presence of effective proteins involved in dsRNA uptake and systemic spread, as the members of Insecta apparently lacks the canonical RNA-dependent RNA polymerase (RdRP) activity required for amplification of the siRNA signal [7]. Lepidopteran species, together with other more derived insects such as the dipterans, are believed to be more refractory to systemic RNAi [22], and variable sensitivity to systemic RNAi has been reported among the Lepidoptera [7, 22, 23].

The transcriptional analysis of insects can also shed light on the molecular basis of development. In insects, the control of development, molting and other processes is largely regulated by the balance between the acyclic sesquiterpenoid juvenile hormone $(\mathrm{JH})$, produced at the corpus allatum, and ecdysteroids synthesized at the prothoracic glands [24]. Synthesis of JHs derives from the mevalonate pathway by the combination of isopentyl pyrophosphate (IPP) and dimethylallyl pyrophosphate (DMAP) to form farnesyl pyrophosphate, which is dephosphorylated to farnesol, oxidized to farnesoic acid, methylated to methyl farnesoate, and converted by epoxidation to JH [24]. Degradation of JH mainly occurs by the action of juvenile hormone esterase (JHE) and/or juvenile hormone epoxide hydrolase (JHEH), both of which lead to a decrease in signaling [24]. In the case of ecdysteroids, since arthropods lack the squalene synthase enzyme, synthesis of ecdysteroids depends on an exogenous sterol source derived from the diet, which usually requires dealkylation and a series of hydroxylation steps [24], catalyzed by cytochrome P450 enzymes encoded by the Halloween genes (phantom, disembodied, shadow and shade) [25].

Tuta absoluta Meyrick (Lepidoptera: Gelechiidae), known as the South American tomato leafminer or pinworm, is a major Solanaceae pest, particularly of tomato (Solanum lycopersicum). This multivoltine microlepidoptera may attack all stages of the host plant, but female adults lay eggs preferentially in leaves, where emergent larvae penetrate and feed, forming galleries in the leaf mesophyll and leading to severe damage of photosynthetic tissues. Major losses also derive from the attack of fruits, aggravated by secondary infection with opportunistic pathogens [26]. This Neotropical species, once restricted to America, invaded Europe and Northern Africa in 2006/2007, from where it is spreading at an alarming rate, threatening major tomato exporters such as India, China, the United States and Mexico [27]. Furthermore, insecticide resistance has been reported in $T$. absoluta, making the development of alternative means for control even more urgent [28]. 
In this study, large-scale RNA sequencing (RNA-seq) was conducted to establish a transcriptome profile of six developmental stages of $T$. absoluta, enabling the identification of potential gene targets for knockdown using the RNAi approach. In addition, the core genes of the basic RNAi mechanism were investigated to determine the suitability of gene silencing in T. absoluta. To validate the coverage of the transcriptome, we searched for genes from insect hormone biosynthetic pathways as potential targets for systemic silencing. Furthermore, comparisons of transcript profiles among stages revealed differentially expressed transcripts (DET) at earlier larval instar stages, which allowed identification of potential gene targets. Five such genes were evaluated using in vitro transcribed dsRNA absorbed by tomato leaflets, fed to $1^{\text {st }}$ instar T. absoluta larvae, resulting in significant reduction of larval body weight while exhibiting knockdown of the genes.

\section{Results}

\section{Transcriptome sequencing and assembling}

Six libraries were generated from $T$. absoluta samples from eggs, adults, and larvae putatively from the $1^{\text {st }}, 2^{\text {nd }}$, $3^{\text {rd }}$, and $4^{\text {th }}$ larval stages. An overall total of $c a .245$ million reads was obtained, ranging from $c a$. 34 to 55 million per library, with an average $44 \%$ GC content (Table 1). In general, all libraries presented good quality, with an average of $94.2 \%$ of reads with base call quality at $99 \%$ probability (Q20) and $87 \%$ at $99.9 \%(\mathrm{Q} 30)$ (Table 1). The sequences were filtered for adaptors and sequencing artifacts, reducing the number of reads per library by 5 to $15 \%$ (filtered reads; Table 1), before transcriptome assembly. The absence of a reference genome for T. absoluta, together with the high coverage in sequenced RNA libraries, led us to the use of a de novo transcriptome assembly to generate a reference for subsequent analyses. The high quality reads were in silico normalized to reduce sequencing coverage. The normalized data were assembled with a minimum fragment overlap of $35 \mathrm{bp}$ and only contigs longer than $300 \mathrm{bp}$ were included in the assembly. The assembled T. absoluta transcriptome contained 93,477 contigs. The assembled reference transcriptome contained 147,141,189 nucleotides in contigs, with an average size of $1,574 \mathrm{bp}$, an N50 of 2,427 bp and an N95 of $480 \mathrm{bp}$. The data were deposited under a NCBI Bioproject [GenBank:PRJNA291932] and under a Sequence Read Archives accession [GenBank:SRS794929].

\section{Transcriptome annotation}

The assembled $T$. absoluta transcriptome was analyzed for gene ontology by Blastx searches against the NCBI non-redundant protein database (nr) using Blast2Go [29]. From the 93,477 contigs, ca. 55,900 (59.8 \%) presented positive Blast hits, with 19,995 (21.4 \%) successfully annotated by gene ontology. The predominant positive Blast hits identified were from insects, particularly Tribolium castaneum, Bombyx mori, Aedes aegypti, Nasonia vitripennis, Acyrthosiphon pisum, Megachile rotundata, Pediculus humanus, and Culex quinquefasciatus (Fig. 1a). In this pair-wise comparison, our dataset showed the highest level of similarity to the Tribolium genome, with over 8,000 hits. Given that the Lepidoptera $B$. mori was the second most common top-hit species, with over 6,000 hits, we consider this effect to be more a consequence of the completeness of the Tribolium genome [30] rather than representative of biological genomic conservation.

Annotation of the T. absoluta transcriptome by Blast2Go revealed the main GO categories under the 'Biological Processes', 'Molecular Function' and 'Cellular Component' ontologies (Fig. 1b). The most enriched terms for 'Biological Process' were cellular $(20.6 \%$ of total number of sequences), metabolic (18.1\%) and single organism processes $(13.5 \%)$, while the dominant categories for 'Molecular Function' were binding (39.2 \%), catalytic (38.8 \%) and transporter activity (7.4 \%) (Fig. 1b). Under 'Cellular Component', cell comprised $34.5 \%$ of the sequences, organelle $22.7 \%$ and membrane $19.6 \%$ (Fig. 1b).

Table 1 Sequenced libraries for each stage of development with total number of generated bases, total of reads per library, percent $\mathrm{G}+\mathrm{C}$, percent of reads with quality above Q20 or above Q30

\begin{tabular}{llllllll}
\hline Libraries & Total bases & $(\mathrm{G}+\mathrm{C}) \%$ & Q20 (\%) & Q30 (\%) & Total reads & Filtered reads & Assembled \\
\hline Eggs & $5,626,900,284$ & 43.53 & 94.4 & 87.4 & $55,711,884$ & $52,617,684$ & $19,261,127$ \\
$1^{\text {st }}$ Stage & $3,718,827,070$ & 41.23 & 94.8 & 88.0 & $36,820,070$ & $28,657,483$ & $12,957,321$ \\
$2^{\text {nd }}$ Stage & $3,497,118,738$ & 43.91 & 94.2 & 87.0 & $34,624,938$ & $32,621,354$ & $12,777,709$ \\
$3^{\text {rd }}$ Stage & $4,469,182,734$ & 45.21 & 93.9 & 86.4 & $44,249,334$ & $41,546,244$ & $13,442,005$ \\
$4^{\text {th }}$ Stage & $3,759,123,848$ & 45.56 & 94.1 & 86.6 & $37,219,048$ & $35,045,786$ & $9,689,430$ \\
Adults & $3,668,050,734$ & 44.01 & 94.0 & 86.5 & $36,317,334$ & $34,105,903$ & $14,378,063$ \\
Total & $24,739,203,408$ & & & & $244,942,608$ & & $82,505,655$ \\
Mean & & 44.0 & 94.2 & 87.0 & & & \\
\hline
\end{tabular}




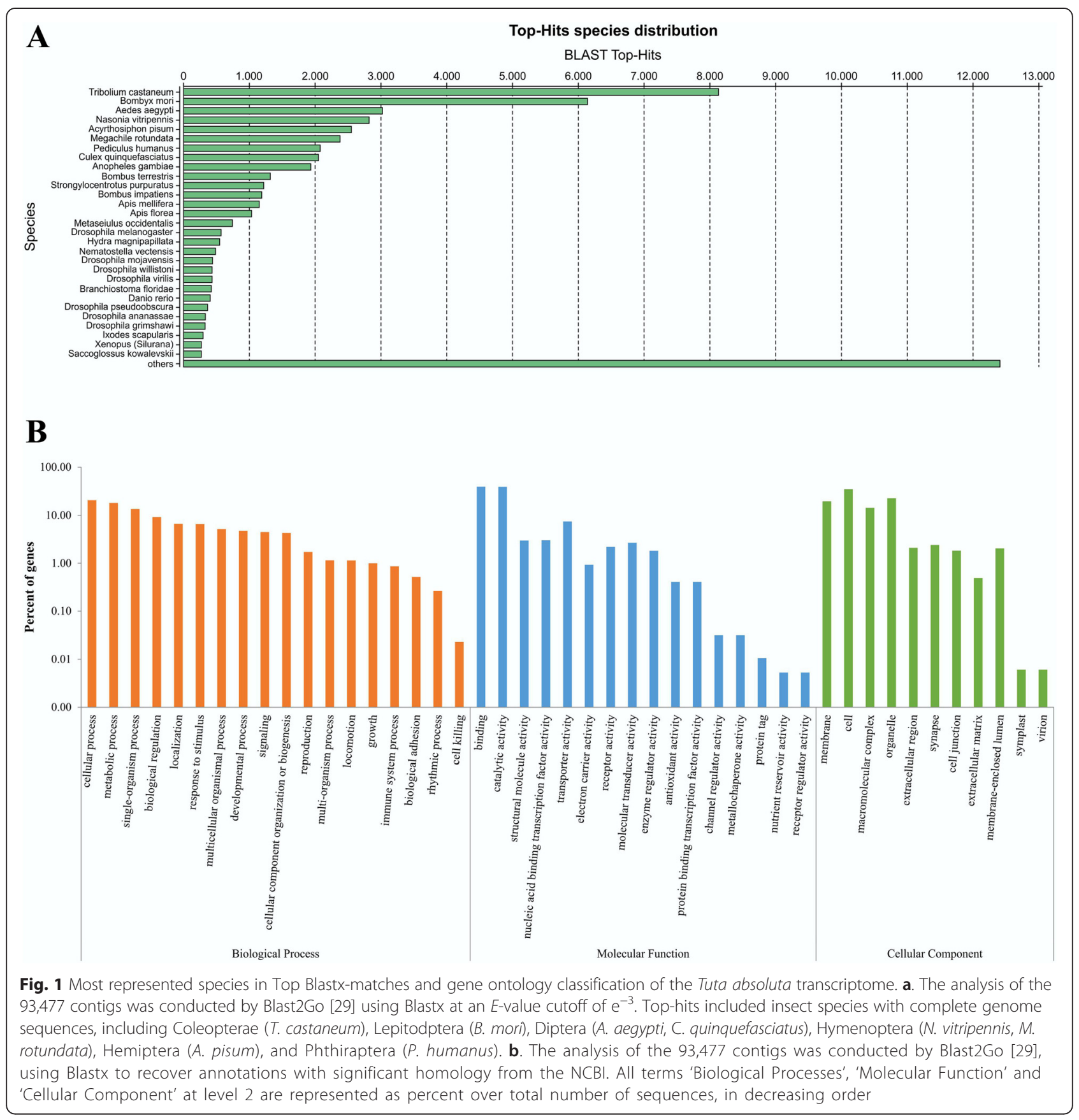

\section{Differential expression during development}

Differential expression between developmental stages of $T$. absoluta was detected by screening genes with $>2$-fold variation with statistical significance. In total, the analysis found 3,917 significant DET during development, with 1,577 DET between eggs and larval stages, 1,128 between larval stages and adults and 1,212 between eggs and adults. No significant difference in expression was detected between the larval stages. Venn diagrams of the
DET among developmental stages allowed the identification of transcripts exclusive or common to the various stages of T. absoluta (Fig. 2). The first Venn diagram comprising DET between eggs and all analyzed larval stages identified 547, 948, 1,087 and 1,201 DET between eggs and the $1^{\text {st }}, 2^{\text {nd }}, 3^{\text {rd }}$ and $4^{\text {th }}$ stages, respectively (Fig. 2a). Comparing larval stages with eggs, we identified 411 DET shared among all four stages analyzed (Fig. 2a). Of the 547 DET between eggs and $1^{\text {st }}$ stage larvae, 52 were exclusive, 


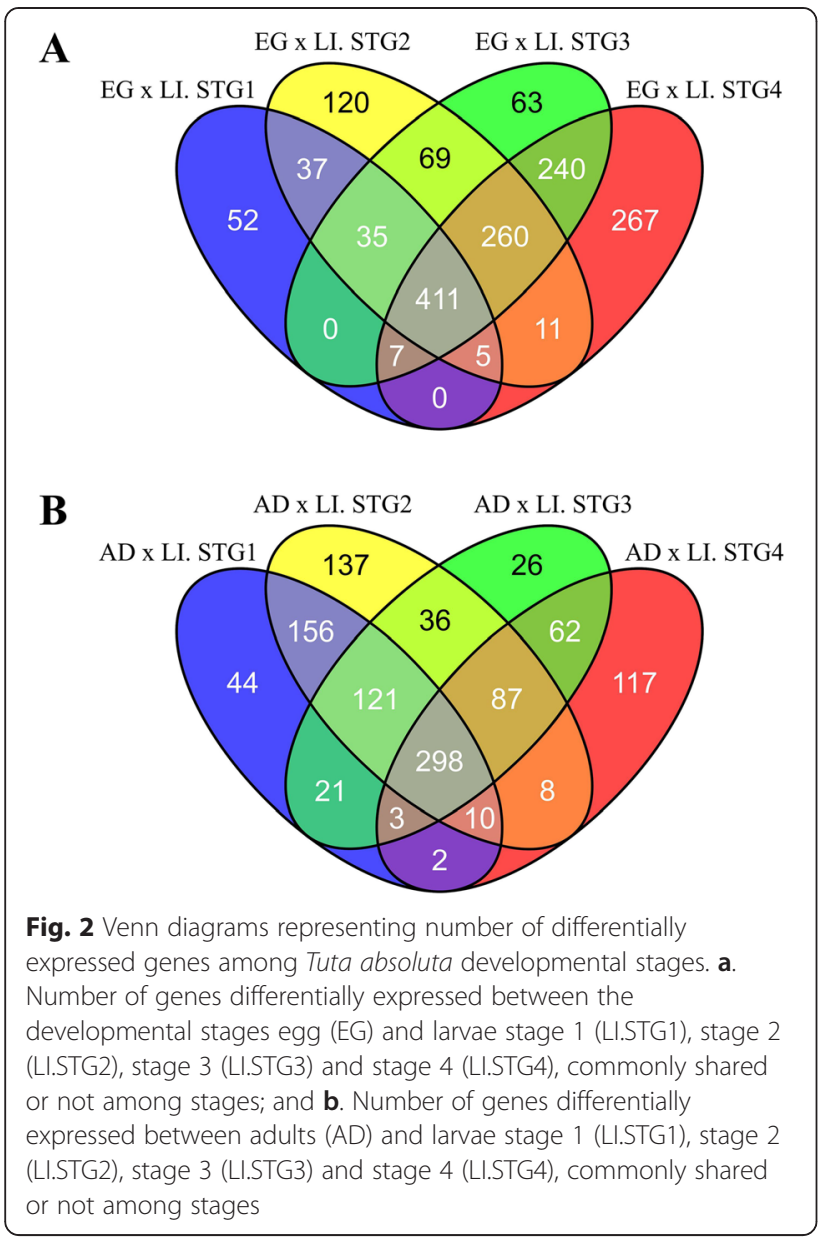

whereas 37 DET were shared between $1^{\text {st }}$ and $2^{\text {nd }}$ stage larvae, and the remaining DET were shared among the three larval stages. Of the 948 DET between eggs and $2^{\text {nd }}$ stage larvae, 120 were exclusive, 69 were shared between the $2^{\text {nd }}$ and $3^{\text {rd }}$ stages, and 11 were shared between the $2^{\text {nd }}$ and $4^{\text {th }}$ stages. Moreover, 63 DET were exclusive to the $3^{\text {rd }}$ stage in comparison with eggs, while 267 were exclusive to the $4^{\text {th }}$ stage (Fig. 2a).

A Venn diagram of DET between adults and larval stages showed that there were 655,853, 654 and 587 DET between adults and the $1^{\text {st }}, 2^{\text {nd }}, 3^{\text {rd }}$ and $4^{\text {th }}$ stages, respectively (Fig. 2b). In this case, 298 DET were commonly found between adults and all the larval stages (Fig. 2b). Of the 655 DET between adults and the $1^{\text {st }}$ stage, 44 were exclusive, 156 were differentially expressed between adults and the $1^{\text {st }}$ and $2^{\text {nd }}$ stage larvae, 21 were shared between $1^{\text {st }}$ and $3^{\text {rd }}$ stage larvae; and two were shared between the $1^{\text {st }}$ and $4^{\text {th }}$ stages (Fig. $2 b$ ). Of the 853 DET between adults and $2^{\text {nd }}$ stage larvae, 137 were exclusive, and 36 were also differentially expressed between adults and $3^{\text {rd }}$ stage larvae. Of the 654 DET between eggs and $3^{\text {rd }}$ larval stage, 26 were exclusive (Fig. 2b). Among the 587 DET between adults and $4^{\text {th }}$ larval stage, 117 were exclusive to this stage (Fig. 2b).

The normalized gene expression values from all the libraries were then used to estimate a Euclidian distance matrix based on transcript profiles to generate a dendrogram and a heatmap describing the similarities among the developmental stages of T. absoluta (Fig. 3). The heatmap and the dendrogram clearly indicated a similarity gradient between the developmental stages, with the transcript profile from $1^{\text {st }}$ stage larvae more similar to that of $2^{\text {nd }}$ stage larvae, and from the $3^{\text {rd }}$ stage more similar to the $4^{\text {th }}$. The gene expression profile from eggs was the most distinct from all the other stages, particularly from adults. There was less similarity among larvae

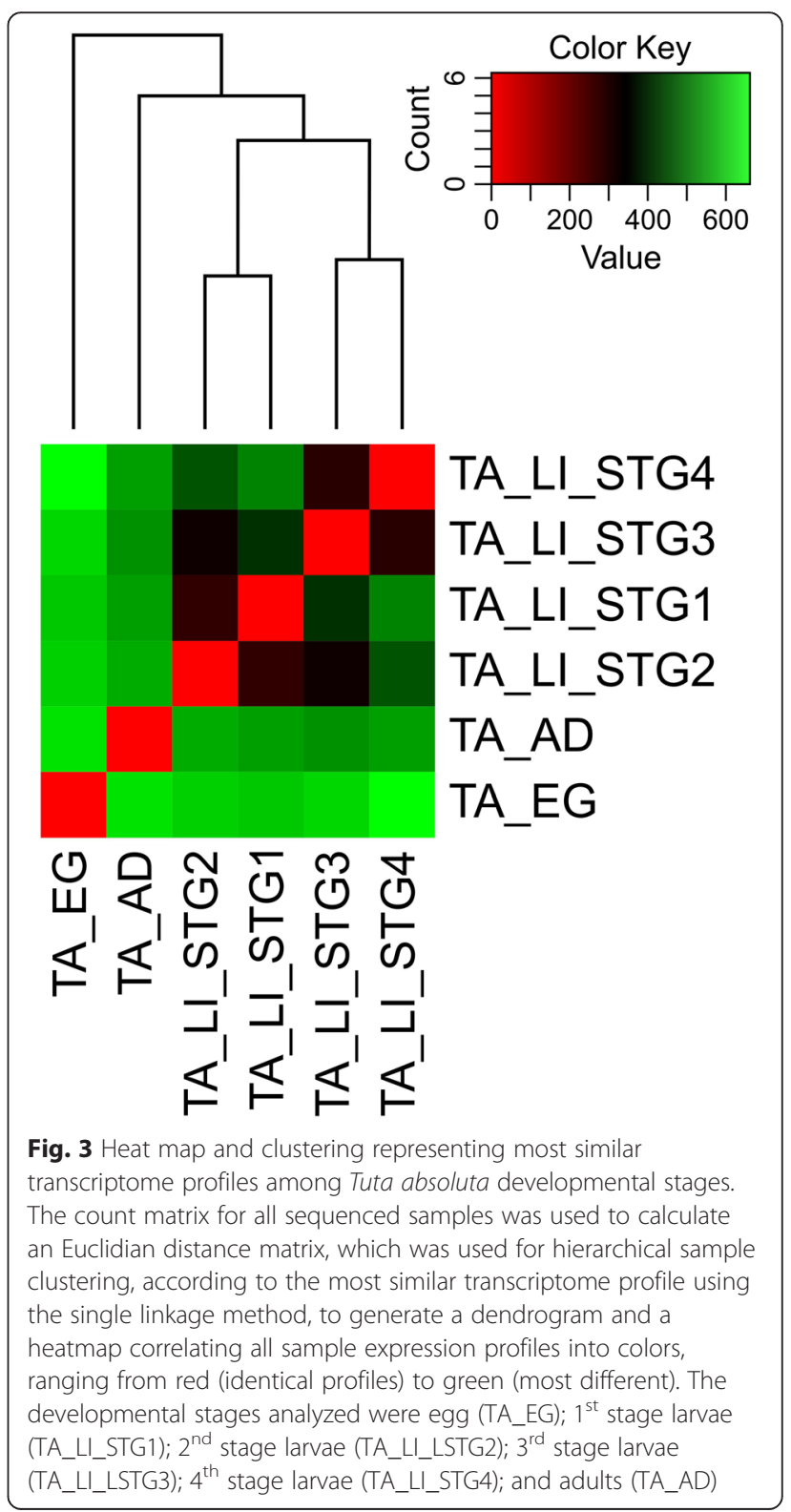


and eggs than for larvae and adults. Among the larval stages, the closer the stages, the higher the similarity of transcripts profiles (Fig. 3).

Annotation of the DET between the developmental stages by Blast2Go revealed that the most enriched terms for the 'Biological Process' ontology were related to metabolic process and single-organism process (Additional file 1: Table S1). When comparing larval stages with the adults, the absolute number of 'Biological Process' terms decreased with development, while the opposite was observed for the larval stages when compared to the egg (Additional file 1: Table S1). A progressive decrease in genes involved with response to stimulus was detected over developing larvae in comparison to adults. In the case of 'Molecular Function', the two most frequent functions among the DET were catalytic activity and binding for all stage comparisons, while transporter activity was more prominent in comparisons between the egg and the larval stages (Additional file 2: Table S2). Under 'Cellular Component' ontology, cell, organelle, macromolecule complex and membrane were the most frequent compartments found for DET genes between eggs and larval stages, but they were less represented (in absolute numbers), or even absent, when larval stages were compared to adults (Additional file 3: Table S3).

The DET were also subjected to a KEGG-based metabolic pathway analysis using Blast2GO. Comparing eggs with the four larval stages, there was an increase in the number of biological pathways related to the DET, from two pathways in the egg versus $1^{\text {st }}$ stage larvae comparison to up to 17 pathways in the egg versus $4^{\text {th }}$ stage (Additional file 4: Table S4). When these larval stages were compared to adults, we observed a relative decrease in number of pathways from the earlier ( 8 or 7 pathways) to the later stages ( 3 or 4 pathways) (Additional file 4: Table S4). In the comparison between eggs and the four larval stages, the steroid hormone biosynthesis and tryptophan metabolism pathways appeared to be altered in the four larvae stages (Additional file 4: Table S4). When we performed a similar analysis by contrasting adults with larval stages, the starch and sucrose metabolism, steroid hormone biosynthesis, and galactose metabolism pathways were altered in all larval stages (Additional file 4: Table S4). The steroid hormone biosynthesis pathway was the only one altered in all four larvae instars compared to both adults and eggs.

\section{Validation of differently expressed transcripts (DET) during development using RT-qPCR}

From the 411 DET between eggs and common to all larval stages (Fig. 2), the 37 DET that were differentially expressed between eggs and the $1^{\text {st }}$ and $2^{\text {nd }}$ larval stages only, and the 298 DET between adults and common to all larval stages, we chose 23 DET to validate the RNA-seq data: 15 among the 411 DET (contigs 10806, 11301, 12524, 12828, 13135, 16411, 16428, 17745, 20172, 21584, $23824,38086,50455,55173$, and 6681), five among the 37 (contigs 2406, 36279, 58512, 75835, and 81147), and three among the 298 DET (contigs 26572, 36206, and 77615) between adults and common to all larval stages. Differences in gene expression between developmental stages were estimated by RT-qPCR using gene-specific primers (Additional file 5: Table S5), with three biological replicates, normalized to three gene references (Rpl5, Rpl23A and $r R N A$; Additional file 5: Table S5).

Of the 23 transcripts evaluated, 22 (95.6\%) were differently expressed between the distinct libraries, with only one contig (12524) showing no significant difference (not shown). Figure 4 illustrates the relative expression results of four DET (58512, 75835, 77615, and 81147 ) by RT-qPCR and RNA-seq, while the remaining 18 are presented on Additional file 6: Figure S1. Among the 22 transcripts differently expressed by RT-PCR, eight (2406, 12828, 21584, 23824, 36279, 58512, 77615 and 81147) presented a highly similar pattern of transcript accumulation to the one derived from the RNA-seq data, with comparable fold-change (Fig. 4; Additional file 6: Figure S1). For the contigs 11301, 20172, 36206, 38086, 50455 and 75835 , the fold-change values were different, but transcript accumulation evaluated by RT-qPCR displayed a similar pattern to the RNA-seq analysis (Fig. 4; Additional file 6: Figure S1). For the remaining eight transcripts, the changes in transcript accumulation detected by RT-qPCR were not related to the pattern observed for the RNA-seq data, but there was clear differential expression between stages (Fig. 4; Additional file 6: Figure S1).

\section{Search for genes involved in the RNA interference mechanism}

The T. absoluta transcriptome dataset was queried for the basic set of genes of the RNAi machinery using homologous genes from Bombyx mori, Drosophila and Caenorhabditis elegans. The main functional steps of siRNA and microRNA processing investigated for homologues included dsRNA uptake by SID-1 and SID-2, dsRNA cleavage into siRNA by Dicer, RISC assembly including putative helicases (Armitage, SpindleE, Rm62) and dsRNA binding (R2D2 and Loquacious), target degradation by endonuclease activity (Argonaute family, Aubergine), and signal amplification by RNA-dependent RNA polymerase (RdRP) (Fig. 5; full list in Additional file 7: Table S6). In this analysis, we identified homologues for sid-1-1 (4 contigs), sid-1-2 (10 contigs), and sid-1-3 (6 contigs), which together represented 11 distinct contigs. We could not find, however, homologous sequences for sid-2. Additionally, we searched for 

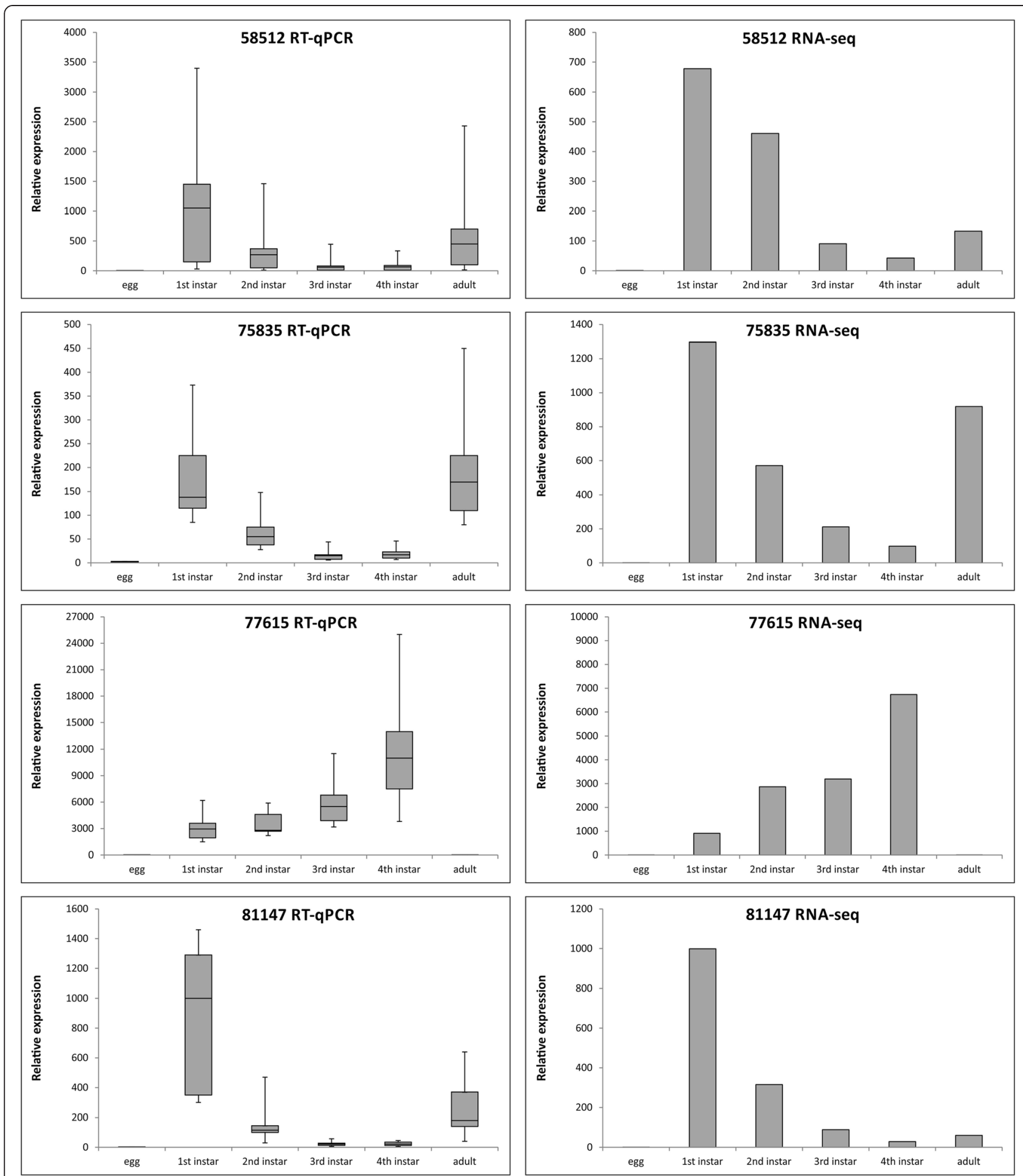

Fig. 4 Comparison of relative gene expression based on RT-qPCR or RNA-seq among developmental stages. Based on the RNA-seq data, 23 contigs with significant differential expression between developmental stages (egg, larval and adults) were chosen to be validated by RT-qPCR. Relative expression of 4 genes (58512; 75835; 77615; and 81147; Additional file 5: Table 55) based on RT-qPCR is represented by whiskers-box plots with standard deviations, and RNA-seq data is represented by fold-differences. Whisker-box plots display average values from three biological replicates, and the box contain $50 \%$ of the variation among samples, while the remaining $50 \%$ are divided between the upper quartile (25\%) and the lower quartile (25\%), represented by error bars (whiskers). The other 18 genes are presented on Additional file 6: Figure S1 


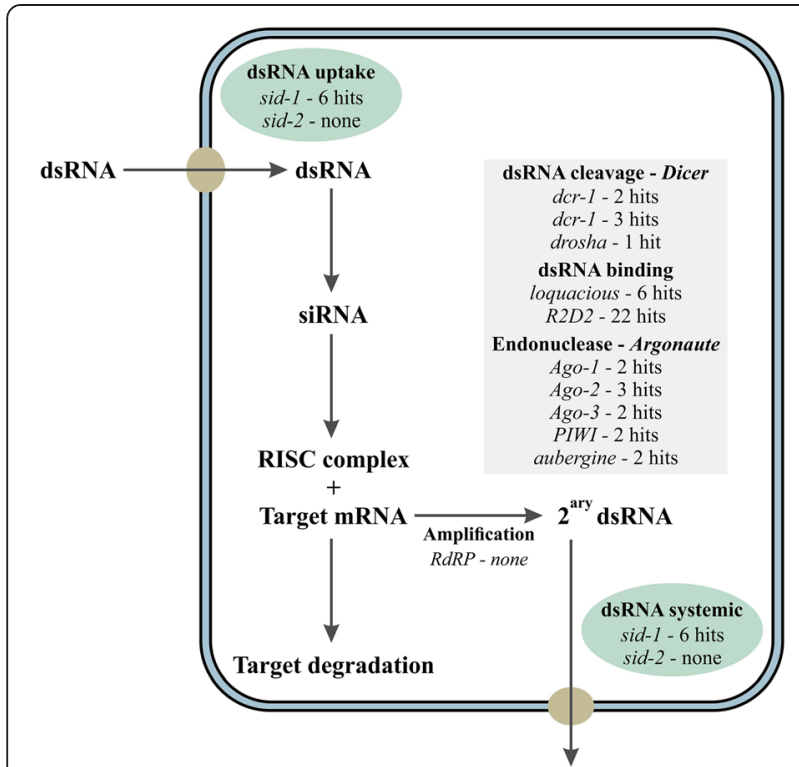

Fig. 5 The core set of genes of RNAi mechanism identified in the Tuta absoluta transcriptome. The analysis of the transcriptome identified genes at minimum $e$-value $<\mathrm{e}^{-30}$ related with the process of RNAi, including dsRNA uptake (sid-1 and sid-2); dsRNA cleavage into siRNA (Dicer or drosha); dsRNA binding (loquacious and R2D2); target degradation by endonuclease activity (argonaute; PIWI; aubergine); and signal amplification by RNA-dependent RNA polymerase $(R d R P)$ with respective number of identified hits at $e<e^{-30}$. Panel adapted from [1]

orthologues of three C. elegans genes ( $r s d-2, r s d-3$, and $r s d-6)$ involved in the germ-line related systemic RNAi response [31], but did not identify any in the T. absoluta transcriptome (Additional file 7: Table S6). Two homologue sequences were found for $d c r-1$, three for $d c r-2$, and one for drosha (Fig. 5). The search for dsRNA binding factors revealed putative homologues for loquacious (6 contigs) and R2D2 (22 contigs), while the search for endonucleases identified homologues for argonaute-1 (two contigs), argonaute-2 (three contigs), argonaute-3 (two contigs), piwi (two contigs), and aubergine (two contigs). No contigs displayed significant similarity with a RNA-dependent RNA Polymerase $(R d R P)$ (Fig. 5). A complete list of the genes associated with the basic mechanism of siRNA and microRNA processing with respective contigs with similarity at an $E$-value $<\mathrm{e}^{-30}$ and per sample FPKM normalized expression values are presented as Additional file 7: Table S6, including those without significant matches. The original results from the Blastx searches are presented as Additional file 8: Table S7.

\section{Search for genes involved in hormone biosynthesis}

A search was performed to identify genes encoding enzymes of the sesquiterpenoid juvenile hormone (JH) and ecdysteroid biosynthetic pathways (Fig. 6). For JH
III biosynthesis, using homologues from B. mori, nine contigs with high similarity $\left(E<\mathrm{e}^{-30}\right)$ to farnesylpyrophosphate synthase genes (Fps, Fpps2, Fpps3) were identified, four to juvenile hormone acid methyltransferase (Jhamt), and four to cytochrome P450 family 15, subfamily A, polypeptide 1 (CYP15A1). For JH III degradation, 20 contigs with similarity to juvenile hormone esterase (JHE) and nine to juvenile hormone epoxide hydrolase (JHEH) were identified (Fig. 6a). All contigs identified, and the respective per sample FPKM normalized expression values are presented as Additional file 9: Table S8. The original results from the Blastx searches are presented as Additional file 10: Table S9.

In the case of ecdysteroid biosynthesis, one contig was identified matching homologues of the Neverland gene (Cholesterol dehydrogenase). Subsequently, contigs with high similarity to cytochrome P450 enzymes encoded by the Halloween genes were detected, with 14 similar to spook (CYP307A), one to phantom (CYP306A1), two to disembodied (CYP302A1), two to shadow (CYP315A1), and two similar to shade (CYP314A1) (Fig. 6b). For ecdysteroid inactivation, four contigs with similarity to a 26-dehydroxylase (CYP18A1), which is putatively responsible for 20,26-Dihydroxyecdysone production, were identified. A complete list of the genes associated with ecdysteroid biosynthesis with respective contigs with similarity at $E<\mathrm{e}^{-30}$ and per sample FPKM normalized expression values are presented as Additional file 9: Table S8. The original results from the Blastx searches are presented as Additional file 10: Table S9.

\section{Gene silencing of novel RNAi candidate genes}

From the transcriptome of T. absoluta, we selected ten genes that were highly expressed in the first larval stages and verified their homology to important genes from other species based on Blastx (Additional file 11: Table S10). The target sequences were then amplified from cDNA derived from larval and pupal stages using specific primers containing the recombination sequences attL1 and attL2 (Additional file 11: Table S10) for later recombination in binary vectors for plant transformation, followed by cloning in pGEM-T. Five sequences were successfully cloned and tested in RNAi assays conducted by providing in vitro transcribed dsRNA in solution to detached tomato leaflets. Together with the novel candidates, the experiment included three other target genes ( $V$-ATPase, $A K, E c R$ ) that were previously evaluated by our group in T. absoluta (Camargo et al., unpublished method), together with a negative control (dsRNA specific for GFP).

A feeding assay was conducted with biological triplicates, with fifty $1^{\text {st }}$ instar larvae feeding on one leaflet. After 5 days feeding on leaflets treated with dsRNA, the larvae were weighed and RNA was extracted to evaluate 
A JUVENILE HORMONE BIOSYNTHESIS

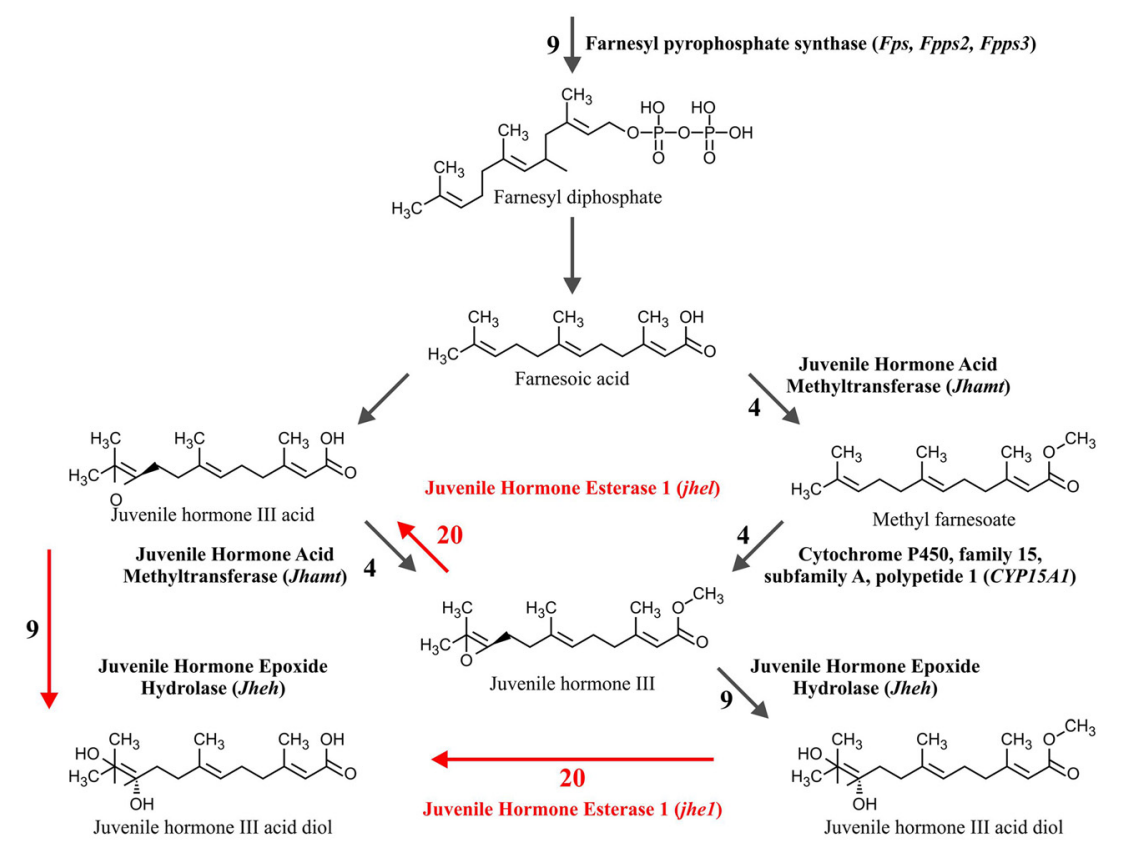

B

ECDYSTEROID HORMONE BIOSYNTHESIS

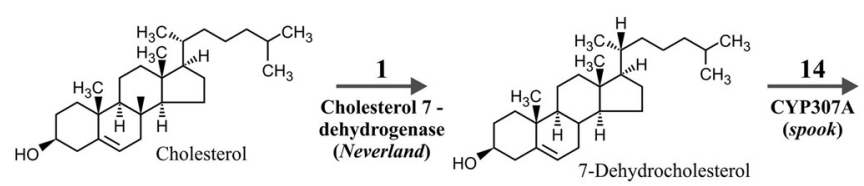

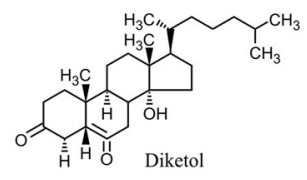

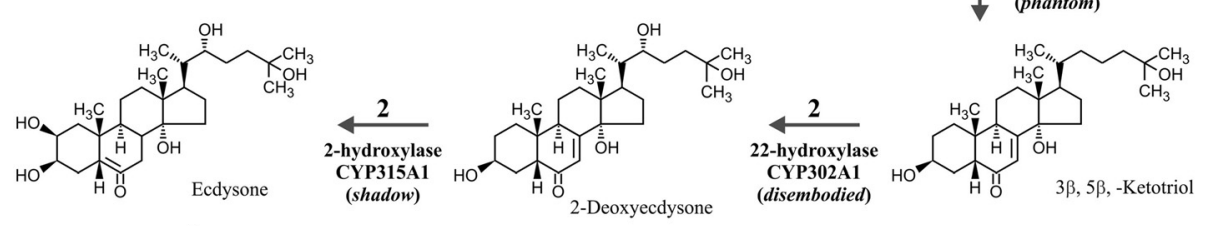

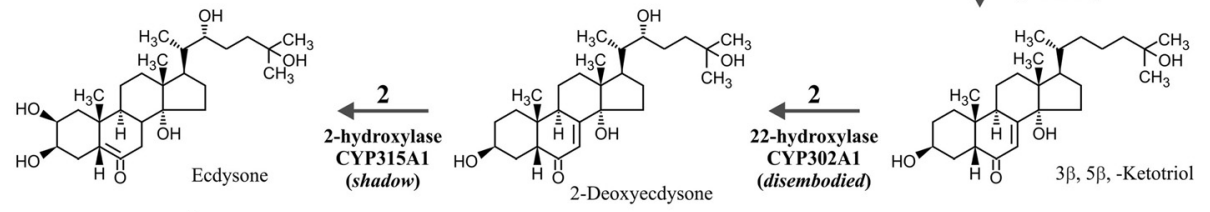

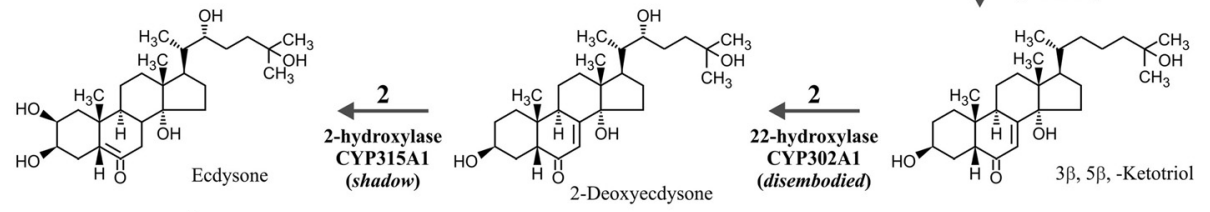

$1 \underset{\begin{array}{c}\text { CYP306A1 } \\ \text { (phantom) }\end{array}}{\text { 25-hydroxylas }}$

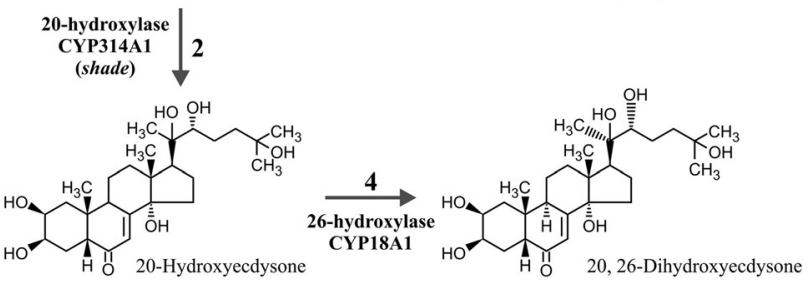

Fig. 6 Juvenile hormone and ecdysteroid hormone biosynthesis with number of hits from the Tuta absoluta transcriptome. $\mathbf{a}$. The analysis of the transcriptome identified homologues genes coding for enzymes of the Juvenile Hormone biosynthetic pathway, including Farnesylpyrophosphate synthase (Fpps); Juvenile Hormone Acid Methyltransferase (Jhamt); and Cytochrome P450 (CYP15A1); and for degradation of the Juvenile Hormone, with Juvenile Hormone Epoxide Hydrolase (Jheh); Juvenile Hormone Esterase 1 (jhe1), with respective number of identified hits at $e<\mathrm{e}^{-30}$ by Blastx. Chemical structures obtained from KEGG. $\mathbf{b}$. The analysis of the transcriptome identified homologues genes coding for enzymes of the Ecdysteoid hormone biosynthetic pathway, including Cholesterol 7-dehydrogenase (Neverland); and a series of cytochrome P450 enzymes encoded by the Halloween genes, such as spook (CYP307A); phantom (CYP306A1); disembodied (CYP302A1); shadow (CYP315A1); and shade (CYP314A1). For ecdysteroid inactivation, a 26-dehydroxylase (CYP18A1), with respective number of identified hits at $e<e^{-30}$ by Blastx. Chemical structures obtained from KEGG 
the effect of RNAi by RT-qPCR (Fig. 7). A significant reduction in larvae weight was detected for all of the genes tested compared with the GFP control, indicating a significant growth delay for the larvae (Fig. 7b). On the other hand, not all genes presented a significant decrease in transcript accumulation (Fig. 7a). The genes $A K$ and the contigs 592 and 1360 did not show a difference in transcript accumulation compared with the GFP control. $A K$ showed the lowest average larvae weight which was not reflected by a reduction in transcript accumulation, and in this case, a large variation in transcript accumulation was observed.

\section{Discussion}

One of the main requisites for developing an RNAimediated pest control strategy is the identification of specific and effective target genes that have a significant impact on insect development or viability, minimizing potential crop losses. Many studies have explored RNAi as a tool to control insects, and over 90 target genes have been evaluated in more than 30 species in eight

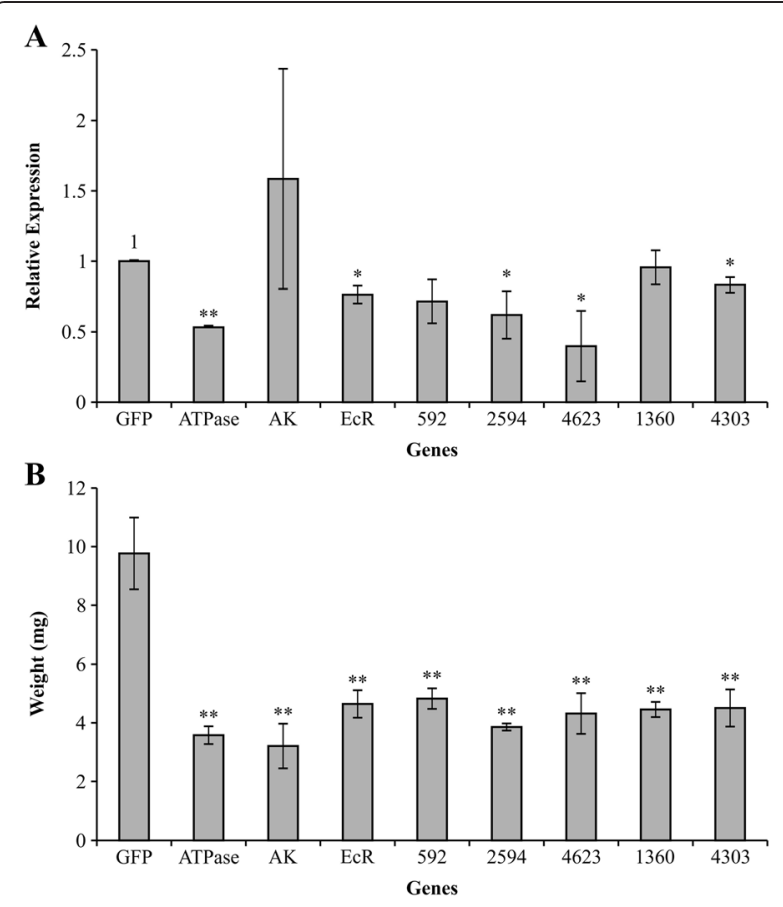

Fig. 7 RNAi effects of 8 genes on Tuta absoluta $1^{\text {st }}$ instar larvae fed in tomato leaflets. a. Relative expression of target genes in larvae, five days after fed on tomato leaflets that absorbed $10 \mu \mathrm{g}$ of dsRNA from each gene target (592; 2594; 4623; 1360; and 4303), and a eGFP control, plus three genes with previous positive results (V-ATPase; AK; ECR; Camargo et al., unpublished method) all in triplicates. Gene expression is expressed in relation to eGFP-fed control. b. Average weight of larvae $(n=30)$ under the same conditions as above. Bars represent standard deviation; ${ }^{* *}$ or ${ }^{1 * * *}$ represent significant at $P<$ 0.05 or $P<0.01$, respectively by T-student test. Gene expression used Rpl5 as gene reference orders (reviewed in [14]). The selection of targets has been based on a candidate gene approach (by orthology to previously described genes) or a screening approach to discover novel target genes [14]. A recurrent problem related with the use of the candidate gene approach is low predictability, with a lack of correspondence of resulting phenotypes, even between close species [18]. One way to identify novel target genes is through genomic information. However, agricultural insect pests are mostly non-model organisms, and little or no genomic information is available for most of them. Recently thought, the development of RNA-seq platforms has enabled the quick assembly of large datasets.

In this study, we were able to build a transcriptome dataset from six developmental stages of T. absoluta based on an overall total of $c a .245$ million reads, assembled into 93,477 contigs with an average size of $1,574 \mathrm{bp}$. The total number of contigs of the reference assembly was higher than expected based on the total number of genes found in Lepidoptera species with full genome sequences available, such as B. mori with 18,501 genes [32] and Plutella xylostella with 18,071 predicted genes [33]. However, de novo transcriptome assembly without a reference genome represents a computational challenge [34, 35], which may result in an imperfect assembly, particularly with short reads [36]. Using the Illumina sequencing platform, comparable results were obtained for the transcriptome of the Asian corn borer (O. furnacalis; Lepidoptera), from which 124,043 contigs were generated with a mean size of $198 \mathrm{bp}$ and an N50 of $211 \mathrm{bp}$, and re-aligned into 79,825 scaffold sequences [16]. For another Lepidoptera, the beet armyworm (S. exigua), a transcriptome was built from pooled samples of all the developmental stages, with over 34 million reads, assembled into 31,414 contigs with an N50 of 542 bp [18]. Similarly, the transcriptome of the feeding canal of the grain aphid (S. avenae; Hemiptera) was assembled into over 83,000 (post-feeding) or 93,000 (pre-feeding) contigs, which were re-aligned into more than 40,000 scaffolds with a mean size of 421-432 bp [17]. Based on the Roche 454-pyrosequencing platform, a cotton ball weevil (A. grandis; Coleoptera) transcriptome was generated with over 500,000 reads, which were assembled into 20,841 contigs with an average size of 676 bp [20]. When directly comparing transcriptome assemblies, the distinct quality parameters adopted in each study must be considered [36]. Nevertheless, our de novo assembly of the T. absoluta transcriptome was comparable in size with previously published assemblies sequenced using the same sequencing platform. Additional quality parameters obtained were the N50 and N95 values, which were $2,427 \mathrm{bp}$ and $480 \mathrm{bp}$, respectively, indicating the formation of large contigs. Additionally, GO annotation showed that the major biological processes, molecular functions, and cellular components were at similar 
proportions to other transcriptomes [18]. Thus, we considered the assembled transcriptome to have achieved our main objective: developing a resource for downstream applications, particularly the use of RNAi in crop protection.

To characterize the RNAi machinery in T. absoluta, we queried the transcriptome for the presence of the core genes involved in RNAi (Fig. 5) using insect or nematode homologues (Additional file 7: Table S6). Although it is a highly conserved cellular mechanism among eukaryotes, some gene homologues responsible for key functions in RNAi are absent in certain taxa. Also, difference responses to RNAi have been reported, particularly among Lepidoptera. Some of the genes involved in the RNAi mechanism, such as ago1 and $d c r 1$, have been used as gene targets for silencing, severely impairing ecdysis in the brown planthopper N. lugens [19].

Most of the expected members of the RNAi core gene set were identified with an $E$-value $<\mathrm{e}^{-30}$, generally with one to three contigs each, which reinforced the quality of the assembled transcriptome (Fig. 5). Some contigs may represent a single gene that could not be assembled under the parameters we set. Lepidopteran species, together with other more derived insects such as the dipterans, are believed to be more refractory to systemic RNAi [22]. One proposed reason for the poor RNAi response in Drosophila is the absence of a canonical 11helix transmembrane channel protein, named SID-1 (systemic RNA interference defective-1), originally identified in C. elegans [31]. Another gene (sid-2), encoding a gut lumen transmembrane protein associated with dsRNA uptake identified in C. elegans, is also absent in Drosophila [1]. Lepidoptera contain three copies of SID1 proteins, which are believed to be involved in dsRNA uptake and systemic spread [19], but there are arguments against this view [31]. In the T. absoluta transcriptome, four contigs showed homology to the sid-1-1 gene of B. mori, 10 contigs to sid-1-2, and six to sid-1-3, but no sid-2 was identified (Fig. 5; Additional file 7: Table S6). Thus, T. absoluta has the appropriate genes for dsRNA uptake and systemic spread, which is the primary step for effective gene silencing. A proposed alternative mechanism of systemic RNAi in Drosophila involves receptor-mediated endocytosis dsRNA uptake [37], and 23 orthologous genes have been identified in N. lugens [19].

Similar to Drosophila, two Dicer paralogues (Dcr-1 and $D c r-2)$ recognized to differentially process miRNA precursors $(D c r-1)$ or long dsRNA (Dcr-2) [21] were identified in the transcriptome of T. absoluta. Drosha, which showed a single highly significant matched contig, shares similar functional features with Dicer but processes miRNA precursors in the nucleus. $R 2 D 2$, loquacious and pasha contain a dsRNA-binding domain
(dsRBD), mediate dsRNA binding to the RISC complex and are considered co-factors of Dicer (loquacious as a co-factor of DCR-1 and R2D2 of DCR-2) and Drosha (Pasha) [19]. We identified 22 contigs homologous to R2D2 and six to loquacious, but only one to pasha that was below our cut-off value (Additional file 7: Table S6; Fig. 5). From the Argonaute (Ago) protein family associated with small RNA identification and binding, and target cleavage, we identified Ago-1, Ago-2, and Ago-3, PIWI, and Aubergine, all based on homologues from $B$. mori (Fig. 5; Additional file 7: Table S6). Ago-1 and Ago-2 are involved in microRNA and siRNA pathways, respectively, while Ago-3, PIWI and Aubergine are associated with piRNA [19]. Furthermore, we searched for a secondary Argonaute family from C. elegans, including PPW-1, PPW-2, Sago1, and Sago2, in our dataset (Additional file 7: Table S6), but no homologous sequences were found. This result is similar to what was reported for $N$. lugens [19]. We also identified putative helicases, such as the Asp spindle and Rm62, but not armitage or tudor sn nucleases (Additional file 7 : Table S6). As previously described in other insects, no RNA-dependent RNA Polymerase $(R d R P)$ was detected, even at low significance $\left(E<\mathrm{e}^{-3}\right)$. According to the homologous genes identified, T. absoluta follows the pattern of other Lepidoptera species, with the RNAi machinery available for successful RNAi, even considering the lack of RdRP. Lepidoptera species are recognized to be recalcitrant to systemic RNAi [22], and variable sensitivity to systemic RNAi has been reported among the Lepidoptera, but the causes for this are still undefined $[7,22,23]$.

Similarly, we were able to identify all the genes encoding enzymes of the sesquiterpenoid juvenile hormone and ecdysteroid biosynthetic pathways as potential targets for RNAi silencing (Fig. 6). In the JH III biosynthesis pathway, there were 4-9 contigs homologous to each gene from $B$. mori, while 9-20 contigs were identified for the degrading enzymes JHE and JHEH (Additional file 9: Table S8). The biosynthesis of ecdysteroid from sterols obtained from the diet depends on the successive action of a dehydrogenase (Neverland), followed by a series of cytochrome P450 enzymes encoded by the Halloween genes. All of these genes were identified in our dataset, matching mostly with one or two contigs, except for spook, with 14 contig hits (Fig. 6). As observed for genes of the RNAi machinery, individual detailed analysis of each of these contigs may reveal a single or few copies for each gene, which may derive from the parameters defined in the assembly process. Interfering with hormone biosynthesis would be an attractive RNAi approach to affect insect development $[38,39]$, if a systemic effect is feasible in T. absoluta since the two major classes of 
hormones are synthesized away from the midgut, where ingested dsRNA reach.

Major losses from T. absoluta infestation derive from galleries formed during larval herbivory [28]. Therefore, we prioritized genes that were more expressed and potentially more relevant at the first larval stages to develop an RNAi approach that would reduce the impact of insect attack. However, it is important to mention that other genes could have an effect on the pest population density. Of the overall 3,917 significantly DET, 1,577 were differentially expressed between eggs and all larval stages and 1,128 between larval stages and adults. Of these DET, 411 were differentially expressed between eggs and common to all larval stages, and another 37 were differentially expressed between eggs and the $1^{\text {st }}$ and $2^{\text {nd }}$ larval stages only. Another 298 were differentially expressed between adults and common to all larval stages (Fig. 2). To validate these differences in expression, we chose 23 DET: 15 among the 411 DET (contigs $10806,11301,12524,12828,13135,16411,16428$, 17745, 20172, 21584, 23824, 38086, 50455, 55173, and 6681), five among the 37 (contigs 2406, 36279, 58512, 75835, and 81147), and three among the 298 DET (contigs 26572, 36206, and 77615) between adults and common to all larval stages. Differences in expression between the developmental stages tested were validated for all genes analyzed, except for one contig (12524). The DET between eggs and the $1^{\text {st }}$ and $2^{\text {nd }}$ larval stages $(2406,36279,58512,75835,81147)$ clearly showed greater expression at the first two stages from the RTqPCR data, corroborating the RNA-seq read count (Fig. 4; Additional file 6: Figure S1), demonstrating that the differences detected among libraries were bona fide. In general, the expression profiles among developmental stages were highly analogous, even for the fold-change values, between the RT-qPCR and RNA-seq data for eight contigs (2406, 12828, 21584, 23824, 36279, 58512, 77615 and 81147). For six contigs (11301, 20172, 36206, 38086, 50455 and 75835), the trend in expression, but not the fold change, was greatly similar. For the remaining eight contigs, there were differences in expression patterns among developmental stages, but there were definite differences in expression between eggs and larvae. Thus, the significant differential expression between stages, validated by RT-qPCR, may assist our choice of target genes to focus on those more expressed in the first larval stages.

Notably, no significant difference in gene expression was observed among any of the larval stages analyzed. One of the reasons for this may derive from the mode of larval sampling used to obtain RNA; they were not staged, which might have led to an overlap between larval instars sampled. We prioritized larval size and age instead of instar to center the RNAi effect at the early stages of herbivory. Particularly when comparing the number of DET between eggs or adults and each larval stage, the numbers differed in each comparison. In this case, it appears that besides the sampling bias, the lack of significant differences in transcripts between larval stages might have derived from a lack of statistical power to detect significance among the differences that occurred. The heatmap and cluster analyses showed a close resemblance in transcript profiles among the larval stages, particularly to the immediate previous or subsequent stage on a similarity gradient, which corroborated the potential lack of discrimination between stages during sampling and/or a biological cause. The gene expression profile from eggs was the most distinct from all the other stages, particularly from adults.

To prove the concept of using transcriptome analysis to discover novel RNAi target genes, we performed gene silencing of transcripts that were highly expressed in the first larval stages (Fig. 7). We previously developed an RNAi delivery assay based on feeding recently emerged larvae with detached tomato leaflets that had absorbed aqueous solution containing in vitro transcribed dsRNA of the target genes (Camargo et al., unpublished method). Using this assay, we successfully demonstrated a significant reduction in larvae weight for all the genes tested in comparison with the GFP control, 5 days after the treatments. Three of the five novel genes tested showed a significant decrease in transcript accumulation under the same conditions. However, the decrease in transcript accumulation in T. absoluta was not dramatic, possibly for a number of reasons. One possibility is the criterion we favored for selecting target genes considering that high levels of expression might indicate essentiality. Therefore, it could be that these highly expressed transcripts have a high functional threshold, and a mild reduction in expression would compromise gene function.

\section{Conclusion}

We demonstrated that the transcriptome dataset generated here represents a valuable genomic resource for screening potential gene targets that affect the development or survival of $T$. absoluta larvae. We successfully identified all of the genes we expected to find in this species, indicating we had sufficient transcriptome sequencing coverage. From the assembled transcriptome, we detected core components of the T. absoluta RNAi machinery, enabling the design of dsRNA experiments for gene expression modulation. Finally, five novel genes identified as differentially more expressed at the $1^{\text {st }}$ stage in our analysis were demonstrated to be effective potential RNAi targets by reducing larval weight and can be considered good candidates for use in RNAi-mediated crop protection. 


\section{Methods}

\section{Biological material}

The colony of $T$. absoluta was originally established from two distinct populations provided by the Insect Biology Laboratory, Dept. of Entomology (ESALQ/USP, Piracicaba, SP, Brazil) and from Bayer Crop Science (Uberlândia, MG, Brazil). The colony has been reared in whole tomato plants or detached leaves under laboratory conditions (temperature $25 \pm 2{ }^{\circ} \mathrm{C} ; 60 \pm 10 \%$ relative humidity; $14 \mathrm{~h}$ photoperiod) [40]. The 'Santa-Clara' tomato cultivar was used for $T$. absoluta maintenance and dsRNA uptake experiments.

\section{Tuta absoluta RNA extraction for sequencing}

Total RNA was extracted separately from whole individuals (eggs, adults, and larval bodies at various stages). Larval staging in $T$. absoluta requires the measurement of the cephalic capsule [41], so we putatively categorized individuals by larval length and days post-emergence (DPE) as $1^{\text {st }}$ stage $(0.6-1.7 \mathrm{~mm}$ long; emergence to $3-4$ $\mathrm{DPE}), 2^{\text {nd }}$ stage $\left(1.2-3.0 \mathrm{~mm}\right.$ long; 5-6 DPE), $3^{\text {rd }}$ stage (2.1-5.0 $\mathrm{mm}$ long; 7-8 DPE) and $4^{\text {th }}$ stage $(3.9-8.0 \mathrm{~mm}$ long; 10-11 DPE). To obtain approximately 50-100 mg of fresh weight, RNA was extracted from $c a$. 1,000 eggs; ca. 2,000 individuals of the $1^{\text {st }}$ instar; ca. 500 individuals of $2^{\text {nd }}$; $c a .100$ individuals of $3^{\text {rd }}$; $c a .30$ individuals of the $4^{\text {th }}$; and ca. 20 adults, using TRIzol (Invitrogen, Carlsbad, CA, USA). An average of $10 \mu \mathrm{g}$ total RNA from each developmental stage was sent on dry ice to Macrogen (Seoul, South Korea) for library construction and sequencing. Libraries were constructed using the TruSeq RNA Sample Prep kit (Illumina, San Diego, CA, USA) and sequenced using a HiSeq 2000 (Illumina) to generate inward paired-end reads of $100 \mathrm{bp}$.

\section{Data processing, transcriptome assembly and annotation} The absence of a reference genome for T. absoluta, together with a high coverage of sequenced RNA libraries, led to the use of a de novo transcriptome assembly pipeline. Initially, raw RNA-seq data were trimmed of adaptors and sequencing artifacts, as well as low quality fragments, with the NGS QC Toolkit [42]. High-quality reads were then subjected to in silico normalization prior to de novo assembly, to reduce the sequencing coverage of highly represented regions with a fragment density higher than $30 \times$. This step was performed to reduce computational complexity without affecting the quality of the assembled transcriptome [35]. Using the de novo transcriptome assembler tool from Trinity [35], the normalized data were assembled with a minimum fragment overlap of $35 \mathrm{bp}$. Only contigs longer than $300 \mathrm{bp}$ were included in the assembled T. absoluta transcriptome for further analyses, including gene ontology (GO) sequence annotation using Blast2Go [29] and
KAAS (KEGG Automatic Annotation Server) [43] submission.

\section{Analysis of differentially expressed transcripts (DET) through developmental stages}

Using the assembled transcriptome as a reference for $T$. absoluta, the sequenced filtered libraries, prior to in silico data normalization, were subjected to transcriptome expression analysis. The filtering coverage previously applied was not used at this stage to avoid interference with the read density when examining gene expression variation. The libraries were mapped to the reference transcriptome using Bowtie with default parameters [44]. Using a read-count methodology, the absolute number of mapped reads for each transcript was represented in a count matrix, with rows representing transcripts and columns representing fragment counts for a specific sample. We then applied a negative binomial distribution method for gene expression analysis with statistical significance. To control false-positives among the DET, a False Discovery Rate (FDR) correction [45] with a cut-off $p$-value $<0.05$ was applied over the calculated statistical significance. To perform this analysis, we included in our pipeline the $\mathrm{R}$ Bioconductor package DESeq [46]. Differential expression between developmental stages was screened by detecting genes with statistical significance. A list of differentially expressed or constitutive transcripts for each stage was produced and analyzed by Blast2GO for gene ontology annotations [29]. Quantitative differences in DET between the developmental stages were represented with Venn diagrams to identify common or exclusive genes among stages. The count matrix for all sequenced samples was also used to calculate a Euclidian distance matrix, which was used for hierarchical sample clustering. According to the most similar transcriptome profile calculated by a single linkage method, a dendrogram and a heatmap were generated, correlating sample expression profiles into colors ranging from red (identical profiles) to green (the most different profiles).

\section{Validation of differences in gene expression among developmental stages by quantitative amplification of reversed transcripts (RT-qPCR)}

To validate differential expression among the developmental stages, 23 genes (Additional file 5: Table S5) were chosen for expression analysis by RT-qPCR. Total RNA from three biological replicates for each developmental stage (eggs, $1^{\text {sr }}, 2^{\text {nd }}, 3^{\text {rd }}$, and $4^{\text {th }}$ larval stages, and adults) was used to produce cDNA. Around $1 \mu \mathrm{g}$ of total RNA was treated with DNase I and 20 U Ribolock (Fermentas, Burlington, Canada) at $37{ }^{\circ} \mathrm{C}$ for $30 \mathrm{~min}$, with the reaction stopped by adding EDTA $(50 \mathrm{mM})$ and heating to $65{ }^{\circ} \mathrm{C}$ for $10 \mathrm{~min}$. One microgram DNAse-treated RNA 
samples were reversed transcribed in $20 \mu \mathrm{L}$ reactions, containing $500 \mu \mathrm{M}$ of each dNTP, $2.5 \mu \mathrm{M}$ oligo dT, $5 \mathrm{mM} \mathrm{DTT}$ and $200 \mathrm{U}$ Revertaid (Fermentas) in appropriate buffer at $50{ }^{\circ} \mathrm{C}$ for $30 \mathrm{~min}$, followed by enzyme inactivation at $85{ }^{\circ} \mathrm{C}$ for $5 \mathrm{~min}$. RT-qPCR reactions contained ca. $40 \mathrm{ng}$ sample cDNA, $5 \mu \mathrm{L}$ Fast SYBR Green Master (Invitrogen), and $0.2 \mu \mathrm{M}$ of each genespecific primer (Additional file 5: Table S5) in a $10 \mu \mathrm{L}$ reaction volume. Amplifications were conducted starting at $50{ }^{\circ} \mathrm{C}$ for $10 \mathrm{~min}$ and $95^{\circ} \mathrm{C}$ for $2 \mathrm{~min}$, followed by 40 three-step cycles of $95{ }^{\circ} \mathrm{C}$ for $15 \mathrm{~s}, 60-61{ }^{\circ} \mathrm{C}$ for $25 \mathrm{~s}$ and $72{ }^{\circ} \mathrm{C}$ for $30 \mathrm{~s}$ in a Qiagen RotorGene-6000 (Qiagen, Valencia, CA, USA). Melting curves were determined after amplification between $72{ }^{\circ} \mathrm{C}$ and $95{ }^{\circ} \mathrm{C}$. Reactions were conducted with technical triplicates and non-template controls. Primer efficiency was determined using a cDNA pool with serial dilutions $\left(1,10^{-1}, 10^{-2}\right.$ and $\left.10^{-3}\right) . C_{Q}$ values were used to determine differences in expression based on [47]. Gene references were RpL5 (large subunit 5 ribosomal protein), $R p l 23 A$ (large subunit 23A ribosomal protein) and $r R N A$ (Additional file 5: Table S5). Data were analyzed using the software REST (Relative Expression Software Tool) [48].

\section{Searches for genes involved with the RNA interference mechanism and hormone biosynthesis}

A list of genes encoding proteins associated with the RNAi mechanism was built based on the literature and Bombyx mori gene sequences were queried against the T. absoluta assembled transcriptome using Blastx with significant $E$-value $<\mathrm{e}^{-30}$. Similarly, genes from the juvenile hormone and ecdysteroid biosynthetic pathways were recovered from KEGG, and homologues from $B$. mori were used to search the T. absoluta transcriptome under similar conditions. RNA interference mechanismand hormone biosynthesis-related transcripts were separately analyzed for each biological condition to show normalized FPKM expression values (Additional file 7: Table S6; Additional file 8: Table S7).

\section{Silencing novel target gene by RNAi}

From the list of transcripts identified by RNA-seq, 10 genes highly expressed at the first larval stage were selected for evaluation as targets for RNAi. Primers were designed for each sequence (Additional file 11: Table S10) with flanking attL1 and attL2 border sequences to enable recombination with binary plasmids using Gateway (Invitrogen) for future use in plant transformation assays. Amplification reactions were conducted in a $20 \mu \mathrm{L}$ volume containing $c a .40 \mathrm{ng}$ cDNA, $3 \mathrm{mM} \mathrm{MgCl}$, $100 \mu \mathrm{M}$ of each dNTP, $0.2 \mu \mathrm{M}$ each primer (Additional file 11: Table S10), and 1.5 U High Fidelity Taq DNA polymerase (Invitrogen) in appropriate buffer. Amplifications were conducted in a Veriti thermocycler (Applied
Biosystems, Foster City, CA, USA) programmed to cycle at $95{ }^{\circ} \mathrm{C}$ for $2 \mathrm{~min}$, followed by 35 cycles of $95{ }^{\circ} \mathrm{C}$ for $30 \mathrm{~s} ; 45-60{ }^{\circ} \mathrm{C}$ for $60 \mathrm{~s} ; 72{ }^{\circ} \mathrm{C}$ for $60 \mathrm{~s}$ according to the target (Additional file 11: Table S10), and a final cycle at $72{ }^{\circ} \mathrm{C}$ for $5 \mathrm{~min}$. The products were electrophoresed in $1 \%$ agarose gels, and the target fragments were excised, purified using a PureLink Quick Gel Extraction Kit (Invitrogen) and cloned into the pGEM-T Easy vector (Promega, Madison, WI, USA) using standard procedures. Identity was confirmed by sequencing three clones of each target gene in an ABI PRISM 3130 (Applied Biosystems).

\section{dsRNA synthesis}

Confirmed clones containing fragments of T. absoluta target genes were used as a template for in vitro transcription reactions to produce dsRNA using T7 RNA polymerase (MegaScript T7, Life Technologies). Target sequences cloned into pGEM-T Easy were amplified using a T7 primer and a SP6 primer fused with a T7 sequence (TAATACGACTCACTATAGGGATTTAGGT GACACTATAG) to operate as a T7 promoter for in vitro transcription in both directions. Template amplification reactions contained $10 \mathrm{ng}$ plasmid DNA, $1.5 \mathrm{mM} \mathrm{MgCl} 2,200 \mu \mathrm{M}$ each dNTP, $0.5 \mu \mathrm{M}$ of each primer, and $1 \mathrm{U}$ Taq polymerase in a $20 \mu \mathrm{L}$ volume. Temperature cycling for amplification started at $95{ }^{\circ} \mathrm{C}$ for $2 \mathrm{~min}$, followed by 35 cycles of $15 \mathrm{~s}$ at $95{ }^{\circ} \mathrm{C}, 20 \mathrm{~s}$ at $60{ }^{\circ} \mathrm{C}, 30 \mathrm{~s}$ at $72{ }^{\circ} \mathrm{C}$, and a final $5 \mathrm{~min}$ at $72{ }^{\circ} \mathrm{C}$. Amplified fragments were run and purified from $1 \%$ agarose gels as above. Purified products were quantified by fluorimetry and used for in vitro transcription in reactions containing $100 \mathrm{ng}$ target DNA, $7.5 \mathrm{mM}$ of each ribonucleotide, and $200 \mathrm{U}$ MegaScript T7 in appropriate buffer in a final volume of $20 \mu \mathrm{L}$. The reactions were conducted at $37{ }^{\circ} \mathrm{C}$ for $16 \mathrm{~h}$, followed by the addition of $2 \mathrm{U}$ DNase for $15 \mathrm{~min}$ at $37{ }^{\circ} \mathrm{C}$. Double-stranded RNA (dsRNA) was purified by precipitation with $7.5 \mathrm{M} \mathrm{LiCl}$ solution $(30 \mu \mathrm{L})$ at $-20{ }^{\circ} \mathrm{C}$ for $1 \mathrm{~h}$, followed by centrifugation at $12,000 \mathrm{~g}$ for $15 \mathrm{~min}$ at $4{ }^{\circ} \mathrm{C}$. The RNA pellet was washed with $70 \%$ ethanol and resuspended in DEPC water. The green fluorescent protein (GFP) gene was used as a negative control. The vector pCAMBIA1302 was used as a template to amplify a negative control gene fragment (276 bp) using the primers GFP-F (TAATACGACTCACTATAGGGCAGTGGAGAGGGTG AA) and GFP-R (TAATACGACTCACTATAGGGTTGA CGAGGGTGTCTC), both containing additional T7 sequences. Similar in vitro transcription was conducted using this template.

\section{RNAi delivery assays to feed $T$. absoluta larvae}

Detached leaves from 'Santa-Clara' tomato had their petioles immersed into $200 \mu \mathrm{L}$ water solution, containing 
$10 \mu \mathrm{g}$ of dsRNA target genes $(592,2594,4623,1360$, and 4303), the GFP control, or three genes with previous positive results ( $V$-ATPase, $A K, E c R$; Camargo et al., unpublished method), all in triplicate. After uptake, 50 individuals at the $1^{\text {st }}$ larval instar were gently placed on the leaves for feeding. Negative controls used dsRNA derived from GFP and every treatment contained three replicates. After 5 days of treatment, 30 larvae were removed and weighed. Total RNA was extracted from these larvae and used to evaluate target gene expression by RT-qPCR.

\section{Availability of supporting data}

The data sets supporting the results of this article are available in the National Center for Biotechnology Information (NCBI) repository (Bioproject PRJNA291932 http://www. ncbi.nlm.nih.gov/bioproject/PRJNA291932/, and Sequence Read Archives SRS794929 http://www.ncbi.nlm.nih.gov/ sra/?term=SRS794929/).

\section{Additional files}

Additional file 1: Table S1. Biological process category annotation by Gene Ontology for differentially expressed transcripts (DET) for pair-wise comparisons between developmental stages of Tuta absoluta by Blast2Go. (PDF 16 kb)

Additional file 2: Table S2. Molecular function category annotation by Gene Ontology for differentially expressed transcripts (DET) for pair-wise comparisons between developmental stages of Tuta absoluta by Blast2Go. (PDF $8 \mathrm{~kb}$ )

Additional file 3: Table S3. Cellular component category annotation by Gene Ontology for differentially expressed transcripts (DET) for pair-wise comparisons between developmental stages of Tuta absoluta by Blast2Go. (PDF $8 \mathrm{~kb}$ )

Additional file 4: Table S4. Kegg-based metabolic pathway annotation for differentially expressed transcripts (DET) for pair-wise comparisons between developmental stages of Tuta absoluta by Blast2Go. (PDF $10 \mathrm{~kb}$ )

Additional file 5: Table S5. Primer sequences of 23 genes differentially expressed between the developmental stages with expected product size, and three gene references used in RT-qPCR to validate differences in read counts among libraries. Annotation is derived from Blast2Go or manual. (PDF 53 kb)

Additional file 6: Figure S1. Comparison of relative gene expression based on RT-qPCR or RNAseq among developmental stages. Based on the RNAseq data, 23 contigs with significant differential expression between developmental stages (egg, larval and adults) were chosen to be validated by RT-qPCR. Relative expression of 18 genes $(2406,6681,10806,11301$, $12828,13135,16411,16428,17745,20172,21584,23824,26572,36206$, 36279, 38086, 50455, and 55173; Additional File 5: Table S5) based on RT-qPCR is represented by whiskers-box plots with standard deviations, and RNAseq data is represented by fold-differences. Whisker-box plots display average values from three biological replicates, and the box contain $50 \%$ of the variation among samples, while the remaining $50 \%$ are divided between the upper quartile (25\%) and the lower quartile (25\%), represented by error bars (whiskers). (PDF 66 kb)

Additional file 7: Table S6. List of genes associated with RNAi mechanism identified in the T. absoluta assembled transcriptome at E-value $<\mathrm{e}^{-30}$ using homologues, particularly from Bombyx mori. FPKM values for each transcript normalized per library is presented. Red rows represent undetected genes within the T. absoluta transcriptome for all sequenced stages, while grey rows are those that did not reach the set $E$-value $<\mathrm{e}^{-30}$. (DOCX 66 kb)

Additional file 8: Table S7. BlastX results with the list of genes associated with RNAi mechanism identified in the T. absoluta assembled transcriptome at $E$-value $<\mathrm{e}^{-30}$ using homologues, particularly from Bombyx mori. (PDF $263 \mathrm{~kb}$ )

Additional file 9: Table S8. List of genes associated with biosynthesis of the two major hormone classes sesquiterpenoid juvenile $(\mathrm{JH})$ and the ecdysteroid hormones identified in the T. absoluta assembled transcriptome at $\mathrm{E}$-value $<\mathrm{e}^{-30}$ using homologues particularly from Bombyx mori. FPKM values for each transcript normalized per library is presented. Red rows represent undetected genes within the T. absoluta transcriptome for all sequenced stages, while grey rows are those that did not reach the set $E$-value $<\mathrm{e}^{-30}$. (PDF $118 \mathrm{~kb}$ )

Additional file 10: Table S9. BlastX results with the list of genes associated with biosynthesis of the two major hormone classes sesquiterpenoid juvenile $(\mathrm{H})$ and the ecdysteroid hormones identified in the T. absoluta assembled transcriptome at E-value $<\mathrm{e}^{-30}$ using homologues particularly from Bombyx mori. (PDF $468 \mathrm{~kb}$ )

Additional file 11: Table S10. Primers used to amplify target-gene fragments flanked by attL1 and attL2 sequences (underlined) for each gene, with expected amplicon size in base pairs (bp) and annealing temperature adopted. (PDF $49 \mathrm{~kb}$ )

\section{Abbreviations}

dsRNA: Double-stranded RNA; RNAi: RNA interference; RISC: RNA-induced silencing complex; RdRP: RNA-dependent RNA polymerase; JH: Juvenile hormone; IPP: Isopentyl pyrophosphate; DMAP: Dimethylallyl pyrophosphate; JHE: Juvenile hormone esterase; JHEH: Juvenile hormone epoxide hydrolase; RNA-seq: Large-scale RNA sequencing; DET: Differentially expressed transcripts; GO: Gene ontology; RT-qPCR: Quantitative amplification reaction of reversed transcripts; SID: Systemic RNA interference defective; Ago: Argonaute; GFP: Green fluorescent protein.

\section{Competing interests}

The authors declare that they have no competing interests.

\section{Authors' contributions}

RAC carried out the laboratory work, developed some of the assays and helped to draft the manuscript. $\mathrm{RHH}$ carried out the bioinformatic analyses. LNS conducted some of the molecular analysis. FMMB helped in the bioinformatic analysis. AF, JEL and HMBS conceived the study, participated in its design and wrote the manuscript. All authors participated in writing and revising, and approved the final manuscript.

\section{Acknowledgments}

This work was financially supported by 'Fundação de Amparo à Pesquisa do Estado de São Paulo - FAPESP' through a Regular Grant (2011/12869-6), and a fellowship to LNS (2012/24990-8) and JEL (2010/11313-1). RAC and FMMB were recipients of CAPES fellowships, and AF received financial support from CNPq. We thank the 'Laboratório Multiusuário de Bioinformática' (LMB) from 'Empresa Brasileira de Pesquisa Agropecuária' (EMBRAPA) by providing computational resources for bioinformatic data analysis. The support by the informatic team from CENA/USP, particularly Gabriel Mendes and George Berner was greatly appreciated. We would like to thank Geraldo Brancalion for his help in preparing the figures.

\section{Author details}

${ }^{1}$ Centro de Energia Nuclear na Agricultura, Universidade de São Paulo, Av. Centenário, 303, CP 96, Piracicaba, SP 13400-970, Brazil. Escola Superior de Agricultura "Luiz de Queiroz" (ESALQ), Universidade de São Paulo, Av. Pádua Dias, 11, CP 09, Piracicaba, SP 13418-900, Brazil. ³ Department of Pediatrics, Cellular and Molecular Medicine, School of Medicine, University of California San Diego, Torrey Pines Scenic Dr, La Jolla, CA 92093-0695, USA. ${ }^{4}$ Graduate Program in Health Science, School of Medicine, Pontifícia Universidade Católica do Paraná, R. Imaculada Conceição, 1155, Prado Velho, Curitiba, PR 80215-901, Brazil. ${ }^{5}$ Instituto de Biologia, Departamento de Histologia e Embriologia, Universidade Estadual de Campinas, R. Charles Darwin, CP 6109, Campinas, SP 13083-863, Brazil. 
Received: 19 December 2014 Accepted: 14 August 2015 Published online: 26 August 2015

\section{References}

1. Price DRG, Gatehouse JA. RNAi-mediated crop protection against insects. Trends in Biotechnol. 2008;26:393-400.

2. Koch A, Kogel KH. New wind in the sails: improving the agronomic value of crop plants through RNAi-mediate gene silencing. Plant Biotechnol J. 2014;12:821-31.

3. Gordon KHJ, Waterhouse PM. RNAi for insect-proof plants. Nature Biotechnol. 2007;25:1231-2

4. Katoch K, Sethi A, Takhur N, Murdock LL. RNAi for insect control: current perspective and future challenges. Appl Biochem Biotech. 2013;171:847-73.

5. Baum JA, Bogaert T, Clinton W, Heck GR, Feldmann P, llagan O, et al. Control of coleopteran insect pests through RNA interference. Nature Biotechnol. 2007:25:1322-6.

6. Mao YB, Cai WJ, Wang JW, Hong GJ, Tao XY, Wang LJ, et al. Silencing a cotton bollworm P450 monooxygenase gene by plant-mediated RNAi impairs larval tolerance of gossypol. Nature Biotechnol. 2007;25:1307-13.

7. Terenius O, Papanicolaou A, Garbutt JS, Eleftherianos I, Huvenne H, Kanginakudru S, et al. RNA interference in Lepidoptera: An overview of successful and unsuccessful studies and implications for experimental design. J Insect Physiol. 2011;57:231-45.

8. Mao YB, Tao XY, Xue XY, Wang $\sqcup$, Chen XY. Cotton plants expressing CYP6AE14 double-stranded RNA show enhanced resistance to bollworms. Transgenic Res. 2011;20:665-73.

9. Zhu JQ, Liu S, Ma Y, Zhang JQ, Qi HS, Wei ZJ, et al. Improvement of pest resistance in transgenic tobacco plants expressing dsRNA of an insect-associated gene ECR. PLoS ONE. 2012;7(6):e38572.

10. Xiong $Y$, Zeng $H$, Zhang $Y, X u$ D, Qiu D. Silencing the HaHR3 gene by transgenic plant-mediated RNAi to disrupt Helicoverpa armigera development. Int J Biol Sci. 2013;9:370-81.

11. Zha WJ, Peng XX, Chen RZ, Du B, Zhu LL, He GC. Knockdown of midgut genes by dsRNA-transgenic plant-mediated RNA interference in the Hemipteran insect Nilaparvata lugens. PLoS ONE. 2011;6(5), e20504.

12. Pitino $M$, Coleman AD, Maffei ME, Ridout CJ, Hogenhout SA. Silencing of aphid genes by dsRNA feeding from plants. PLoS ONE. 2011;6(10), e25709.

13. Xu L, Duan X, LV Y, Zhang X, Nie Z, Xie C, et al. Silencing of an aphid carboxylesterase gene by use of plant-mediates RNAi impairs Sitobion avenae tolerance of Phoxim insecticides. Transgenic Res. 2014;23:389-96.

14. Zhang H, Li HC, Miao XX. Feasibility, limitation and possible solutions of RNAi-based technology for insect pest control. Insect Sci. 2013;20:15-30.

15. Wang Z, Gerstein M, Snyder M. RNA-Seq: a revolutionary tool for transcriptomics. Nature Rev Genet. 2009;10:57-63.

16. Wang Y, Zhang H, Li H, Miao X. Second-generation sequencing supply an effective way to screen RNAi targets in large scale for potential application in pest insect control. PLoS ONE. 2011;6(4), e18644.

17. Zhang M, Zhou Y, Jones HD, Gao Q, Wang D, Ma Y, et al. Identifying potential RNAi targets in grain aphid (Sitobion aveane F.) based on transcriptome profiling of its alimentary canal after feeding on wheat plants. BMC Genomics. 2013;14:560.

18. Li H, Jiang W, Zhang Z, Xing Y, Li F. Transcriptome analysis and screening for potential target genes for RNAi-mediated pest control of the beet armyworm. Spodoptera exigua. PLoS ONE. 2013;8(6):e65931.

19. Xu HJ, Chen T, Ma XF, Xue J, Pan PL, Zhang XC, et al. Genome-wide screening for components of small interfering RNA (siRNA) and micro-RNA (miRNA) pathways in the brown planthopper, Nilaparvata lugens (Hemiptera: Delphacidae). Insect Mol Biol. 2013;22:635-47.

20. Firmino AAP, Fonseca FCA, de Macedo LLP, Coelho RR, de Souza Jr JDA, Togawa RC, et al. Transcriptome analysis in cotton boll weevil (Anthonomus grandis) and RNA interference in insect pests. PLOS ONE. 2013;8(12), e85079.

21. Meister G, Tuschi T. Mechanisms of gene silencing by double stranded RNA. Nature. 2004;431:343-9.

22. Gu L, Knipple DC. Recent advances in RNA interference research in insects: implications for future insect pest management strategies. Crop Prot. 2013:45:36-40
23. Garbutt JS, Bellés X, Richards EH, Reynolds SE. Persistence of double-stranded RNA in insect hemolymph as a potential determiner of RNA interference success: Evidence from Manduca sexta and Blattella germanica. J Insect Physiol. 2013;59:171-8.

24. Klowden MJ. Endocrine systems. In: Klowden MJ, editor. Physiological systems in insects. 2nd ed. Burlington, MA: Academic; 2007. p. 1-49.

25. de Loof A. Ecdysteroids, juvenile hormone and insect neuropeptides: Recent successes and remaining major challenges. Gen Comp Endocr. 2008; 155:3-13.

26. Cifuentes D, Chynoweth R, Bielza P. Genetic study of Mediterranean and South American populations of tomato leafminer Tuta absoluta (Povolny, 1994) (Lepidoptera: Gelechiidae) using ribosomal and mitochondrial markers. Pest Manag Sci. 2011;67:1155-62.

27. Desneux N, Luna MG, Guillemaud T, Urbaneja A. The invasive South American tomato pinworm, Tuta absoluta, continues to spread in Afro-Eurasia and beyond: the new threat to tomato world production. J Pest Sci. 2011;84:403-8.

28. Urbaneja A, Desneux N, Gabarra R, Arno J, González-Cabrear J, Mafra-Neto A, et al. Biology, ecology and management of the South American tomato pinworm, Tuta absoluta. in potential invasive pests of agricultural crops. In: Peña JE, editor. Potential invasive pests of agricultural crops. Oxfordshire: CABI; 2013. p. 98-132.

29. Conesa A, Gotz S, Garcia-Gomez JM, Terol J, Talon M, Robles M. Blast2GO: a universal tool for annotation, visualization and analysis in functional genomics research. Bioinformatics. 2005;21:3674-6.

30. Tribolium Genome Sequencing Consortium. The genome of the model beetle and pest Tribolium castaneum. Nature. 2008;452:949-55.

31. Miyata K, Ramaseshadri P, Zhang Y, Segers G, Bolognesi R, Tomoyasu Y. Establishing an in vivo assay system to identify components involved in environmental RNA interference in the Western Corn Rootworm. PLoS ONE. 2014;9(7), e101661

32. Xia Q, Xia Q, Zhou Z, Lu C, Cheng D, Dai F, et al. A draft sequence for the genome of the domesticated silkworm (Bombyx mori). Science. 2004;306:1937-40.

33. You M, Yue Z, He W, Yang X, Yang G, Xie M, et al. A heterozygous moth genome provides insights into herbivory and detoxification. Nature Genet. 2013;45:220-5

34. Hass BJ, Zody MC. Advancing RNA-seq analysis. Nature Biotechnol. 2010;28:421-3.

35. Grabherr MG, Hass BJ, Yassour M, Levin JZ, Thompson DA, Amit I, et al. Full length transcriptome assembly from RNA-seq data without a reference genome. Nature Biotechnol. 2011;29:644-52.

36. Schliesky S, Gowik U, Weber APM, Bräutigam A. RNA-seq assembly - are we there yet? Frontiers Plant Sci. 2012;3:220.

37. Saleh MC, van Rij RP, Hekele A, Gillis A, Foley E, O'Farrell PH, et al. The endocytic pathway mediates cell entry of dsRNA to induce RNAi silencing. Nat Cell Biol. 2006;8:793-802.

38. Luan JB, Ghanim M, Czosnek H. Silencing the ecdysone synthesis and signaling pathway genes disrupt nymphal development in the whitefly. Insect Biochem Mol Biol. 2013;43:740-6.

39. Asokan R, Sharath Chandra G, Manamohan M, Krishna Kumar NK, Sita T. Response of various target genes to diet-delivered dsRNA mediated RNA interference in the cotton bollworm, Helicoverpa armigera. J Pest Sci. 2014;87:163-72.

40. Pratissolil D, Pezzopanel JEM, Espostil MDD, Bertazol CL, Fornazier JM. Estimativa do número de gerações de Trichogramma pretiosum Riley na traça do tomateiro Tuta absoluta (Meyrick), com base nas exigências térmicas. An Soc Entomol Bras. 1998;27(1):109-15.

41. Giustolini TA, Vendramin JD, Parra JRP. Número de instares larvais de Tuta absoluta (Meyrick) em genótipos de tomateiro. Scientiae Agricola. 2002;59:393-6.

42. Patel RK, Jain M. NGS QC Toolkit: a toolkit for quality control of next generation sequencing data. PLOS ONE. 2012;7(2), e30619.

43. Moriya Y, Itoh M, Okuda S, Yoshizawa A, Kanehisa M. KAAS: an automatic genome annotation and pathway reconstruction server. Nucleic Acids Res. 2007:35:W182-5.

44. Langmead B, Trapnell C, Pop M, Salzberg SL. Ultrafast and memory-efficient alignment of short DNA sequences to the human genome. Genome Biol. 2009;10:R25

45. Benjamini $Y$, Hochberg Y. Controlling the false discovery rate: a practical and powerful approach to multiple testing. J R Stat Soc B. 1995;57:289-300. 
46. Anders $\mathrm{S}$, Huber $\mathrm{H}$. Differential expression analysis for sequence count data. Genome Biol. 2010;11:R106.

47. Livak KJ, Schmittgen TD. Analysis of relative gene expression data using real-time quantitative PCR and the 2(-Delta Delta Ct) method. Methods. 2001;25:402-8

48. Pfaffl MW, Horgan GW, Dempfle L. Relative expression software tool (REST) for group-wise comparison and statistical analysis of relative expression results in real-time PCR. Nucleic Acids Res. 2002;30:1-10.

Submit your next manuscript to BioMed Central and take full advantage of:

- Convenient online submission

- Thorough peer review

- No space constraints or color figure charges

- Immediate publication on acceptance

- Inclusion in PubMed, CAS, Scopus and Google Scholar

- Research which is freely available for redistribution 